\title{
Open-label, phase I dose-escalation study of sodium selenate, a novel activator of PP2A, in patients with castration-resistant prostate cancer
}

\author{
NM Corcoran", , CM Hovens', M Michael' ${ }^{2}$, MA Rosenthal ${ }^{3}$ and AJ Costello' \\ 'Department of Surgery, Division of Urology University of Melbourne, Royal Melbourne Hospital, 5th Floor Clinical Sciences Building, Royal Parade, \\ Parkville, Victoria 3050, Australia; 'Division of Haematology and Medical Oncology, Peter MacCallum Cancer Centre, Locked Bag I, A'Beckett Street, \\ Melbourne, Victoria 3002, Australia; ${ }^{3}$ Department of Medical Oncology, Royal Melbourne Hospital, Grattan Street, Parkville, Victoria 3050, Australia
}

\begin{abstract}
BACKGROUND: Angiogenesis is fundamental to the progression of many solid tumours including prostate cancer. Sodium selenate is a small, water-soluble, orally bioavailable activator of PP2A phosphatase with anti-angiogenic properties.

METHODS: This was a dose-escalation phase I study in men with asymptomatic, chemotherapy-naïve, castration-resistant prostate cancer. The primary objective was to determine the maximum tolerated dose (MTD). Secondary objectives included establishing the safety, tolerability and pharmacokinetic profile.

RESULTS: A total of 19 patients were enrolled. The MTD was $60 \mathrm{mg}$ per day. Dose-limiting toxicity (fatigue and diarrhoea) was observed at $90 \mathrm{mg}$ per day. The most frequently reported treatment-related adverse events across all treatment cohorts were nausea, diarrhoea, fatigue, muscle spasms, alopecia and nail disorders. No grade 4 toxicities were observed and there were no deaths on study. Linear pharmacokinetics was observed. One patient had a PSA response $>50 \%$. Median time to PSA progression (for nonresponders) was 14.2 weeks. Mean PSA doubling time increased during the main treatment phase from 2.18 months before trial to 3.85 months.

CONCLUSION: Sodium selenate is well tolerated at a dose of $60 \mathrm{mg}$ per day with modest single-agent efficacy similar to other anti-angiogenic agents. Further trials in combination with conventional cytotoxic regimens are warranted.

British Journal of Cancer (2010) I 03, 462-468. doi:10.1038/sj.bjc.6605798 www.bjcancer.com
\end{abstract}

Published online 20 July 2010

(c) 2010 Cancer Research UK

Keywords: prostate cancer; sodium selenate; PP2A phosphatase; anti-angiogenesis; phase I study

Prostate carcinoma is a common cancer, which is lethal in up to $15 \%$ of patients (Baade et al, 2004; Jemal et al, 2009). The vast majority of patients die from castrate-resistant disease, evidenced by continued tumour progression despite low levels of circulating androgens. The ability of traditional cytotoxic agents to affect meaningfully on survival is limited, with the most efficacious docetaxel-based regimens extending median survival by just more than 2 months. There is thus an urgent need for new agents that either significantly affect the disease as a stand-alone agent or sensitise tumours to existing therapies.

The ability to independently initiate new blood vessel formation is a 'hallmark' of solid tumours. The importance of angiogenesis in the development and progression of prostate cancer is underscored by numerous studies evaluating microvessel density (MVD), a histological correlate of angiogenic potential, in human prostate samples. The MVD of prostate cancer is consistently higher than normal tissue, and is positively associated with more advanced tumour stage, higher Gleason scores, and the presence of metastatic disease. In addition, MVD predicts biochemical recurrence after both radical prostatectomy and external beam

*Correspondence: Dr NM Corcoran; E-mail: niallmcorcoran@gmail.com Received 12 April 2010; revised 16 June 2010; accepted 18 June 20I0; published online 20 July 2010 radiotherapy (Halvorsen et al, 2000; Revelos et al, 2007), and when used with other markers of angiogenic potential, predicts overall survival (Mucci et al, 2009). Similarly, significantly higher levels of VEGF, the predominant pro-angiogenic signalling molecule, are detected in patients with metastatic disease, and in hormone refractory prostate cancer, when both serum and urine levels predict survival (Bok et al, 2001; George et al, 2001). Over the last two decades the role of protein kinases in co-ordinating cancerrelated neo-angiogenesis through the 'switch-like' reversible phosphorylation of key regulatory molecules in response to proangiogenic signals has been extensively elaborated (Ahmed and Bicknell, 2009; Aragon-Ching and Dahut, 2009). On the basis of this knowledge a number of antibodies and small-molecule kinase inhibitors that target pro-angiogenic signalling have been developed, and have been successfully translated to clinical use in renal, breast, non-small cell lung, colorectal and hepatic carcinoma (Ivy et al, 2009). At the same time, relatively little attention has been paid to the role of protein phosphatases, molecules that promote subsequent dephosphorylation, in limiting or even suppressing pro-angiogenic signalling. We have identified a specific seleniumcontaining compound, sodium selenate, which significantly boosts the activity of a key intracellular phosphatase, PP2A (Corcoran et al, 2010). PP2A has been shown to negatively regulate a number of intracellular proteins that are important in angiogenic signalling, 
particularly components of the PI3K/Akt and MAPK pathways, in endothelial cells (Young et al, 2002; Young, 2004; Kim et al, 2009). We have previously shown that sodium selenate inhibits experimental tumour growth in an orthotopic model of castrationresistant prostate cancer (CRPC) by inhibiting vessel branching (Corcoran et al, 2004). This first-time-in-human study examined the novel PP2A stimulator, sodium selenate, in men with CRPC.

The primary aims of this study were to assess the safety, tolerability and pharmacokinetics of sodium selenate in men with CRPC.

\section{PATIENTS AND METHODS}

\section{Patient selection}

Patients with CRPC defined by at least three successive rises in serum PSA at least 2 weeks apart, in the presence of castrate levels of serum testosterone, were eligible for enrolment in this study. Anti-androgen therapy must have been discontinued at least 4 weeks before entry into the trial, with evidence of continuing PSA rises after this time, with a PSA level $\geqslant 5 \mu \mathrm{gl}^{-1}$ required at study entry. LHRH agonists were continued and allowed concurrently. In addition, patients were required to be asymptomatic or have only minor symptoms due to their prostate cancer, have a WHO performance status $\leqslant 2$, and an estimated life expectancy of at least 6 months. Patients were also required to have adequate renal (serum creatinine $<1.5$ ULRR), liver (serum bilirubin $<1.25$ and AST/ALT <2.5 ULRR) and haematological reserve (absolute neutrophil count $>1 \times 10^{9} 1^{-1}$ and platelets $>100 \times 10^{9} 1^{-1}$ ), and have no evidence of severe or uncontrolled systemic disease.

Patients treated previously with cytotoxic chemotherapy or strontium therapy, or who had coexisting malignancies or malignancies diagnosed within the last 5 years (with the exception of non-melanomatous skin cancer), were ineligible for enrolment. Treatment with any investigational agent within 4 weeks of study entry was not allowed, and patients with unresolved chronic toxicity greater than Common Terminology Criteria grade 2 from previous anti-cancer therapy or incomplete healing from previous surgery were excluded. Although the previous use of commercially available selenium supplementation was not a specific exclusion criterion, concomitant use over the study period was not permitted. Similarly, the concomitant use of unapproved or herbal remedies for prostate cancer was prohibited during the study period.

The study received institutional review board approval and all patients gave written informed consent.

\section{Study design and treatment plan}

This was an open-label phase I dose-escalation study. After signing informed consent, patients underwent baseline testing to confirm eligibility. Patients who satisfied the inclusion and exclusion requirements were invited to enter the study according to the doseescalation criteria. Baseline evaluations were conducted within 2 weeks of starting therapy. Sodium selenate was administered daily for 3 weeks (one cycle). After four cycles of therapy (12 weeks), patients with stable or responding disease, and who wished to continue on study, were offered ongoing treatment for a further 12 weeks. During the main treatment phase (weeks 0-12) patients underwent assessments for safety and tolerability at the end of each cycle. Patients opting to continue the drug beyond this time were similarly evaluated at six weekly intervals. All patients were assessed for safety 28 days after the last dose of study drug, and where possible, all patients were evaluated 3 months after their final treatment with sodium selenate.

At study commencement, sodium selenate was administered as an oral capsule taken once a day on an outpatient basis. Patients were given a fixed dose and there was no intra-patient doseescalation. After the initial pharmacokinetic analysis demonstrated a short drug half-life in serum, the same total daily dose was given in three divided doses in one 24 -h period to generate steady-state plasma selenate levels (beginning with patient 5).

For the initial dose levels, an accelerated titration design was used with one patient at each dose $(5 \mathrm{mg}, 10 \mathrm{mg}, 15 \mathrm{mg}, 30 \mathrm{mg})$ treated and evaluated at the end of 3 weeks. Toxicities were graded according to Common Terminology Criteria for Adverse Events version 3. Drug-related toxicity (DRT) was defined as any grade 2 non-haematological toxicity and/or any grade 3 haematological toxicity. In the absence of a DRT, commencement of a patient at the next dose level occurred. Three patients were subsequently enrolled to each fixed-dose cohort, with planned dose levels of 60 , 90 and $120 \mathrm{mg}$ per day. The protocol was later amended to include $45 \mathrm{mg}$ per day to collect additional safety information. Doseescalation continued until dose-limiting toxicity (DLT) was observed in one-third of patients during cycle 1. The DLT was defined as any grade 3/4 non-haematological toxicity and/or and grade 4 haematological toxicity. If DLT was observed in one of three patients in a cohort, a further three patients were recruited to that dose level. If one further patient experienced DLT, then this defined the DLT level. If no DLT was observed, patients were recruited to the next dose level. The maximum tolerated dose (MTD) was defined as the dose level below that in which $\geqslant 33 \%$ ( 2 of 6 ) of patients were observed to have a DLT. The MTD was then expanded to six patients.

If patients developed $\geqslant$ grade 2 haematological and/or nonhaematological toxicity during the main treatment phase, sodium selenate was not administered. If treatment was deferred because of toxicity, it could be re-initiated once toxicity was $\leqslant$ grade 1 . Treatment delays of up to 2 weeks were allowed, but no dose reductions were permitted. In the absence of treatment delays due to adverse events, treatment continued for up to four cycles or until the disease progressed, the occurrence of an inter-current illness that prevented further administration of treatment, or the patient decided to withdraw from the study. In the absence of toxicity, patients were allowed to remain on selenate beyond the main treatment phase if they chose to do so.

\section{Drug product}

Sodium selenate was blended with the inert pharmaceutical excipient lactose and filled into capsules using a Dott Bonapace (Milan, Italy) semi-automatic capsule filler, under GMP conditions, using the appropriate size capsules. Three dosage strengths were prepared, containing 5,20 or $50 \mathrm{mg}$ of sodium selenate. Independent quality control testing was performed on the finished product, and stability at $25^{\circ} \mathrm{C}$ for the time course of the study confirmed.

\section{Patient evaluation}

Before registration, a complete medical history and physical examination were performed, castrate levels of serum testosterone confirmed, and baseline haematology and biochemistry were obtained. Patients were then evaluated at the start of each treatment cycle for possible adverse events or toxicity (performance status, physical examination, electrocardiogram, full blood count, urea and electrolytes, liver function tests). All patients entering the trial had an additional safety visit at 4 and 12 weeks after completion of therapy to assess for residual toxicity. To assess for tumour response, serum PSA was measured at the completion of each treatment cycle. Although the trial was initiated before the publication of the Prostate Cancer Trials Working Group 2 criteria for PSA end points, PSA data are analysed according to their recommendations (Scher et al, 2008). In particular, the percentage change in PSA from baseline to the 
completion of initial 12-week evaluation, or date of withdrawal from study if earlier, is presented in a waterfall plot. For patients experiencing an initial decline in PSA, time to PSA progression is defined by the date of documentation of PSA $\geqslant 25 \%$ above nadir with an absolute increase of $\geqslant 2 \mathrm{ng} \mathrm{ml}^{-1}$ confirmed on a second reading $\geqslant 3$ weeks later. For patients not experiencing a decline in baseline PSA, the date of progression is defined by the date of documentation of PSA $\geqslant 25 \%$ with an absolute increase $\geqslant 2 \mathrm{ng} \mathrm{ml}^{-1}$ above baseline, after 12 weeks on treatment. In addition, PSA doubling time was calculated using the log-slope method as previously described (Daskivich et al, 2006). For pretrial PSA measurements, a weighted linear regression was performed, with PSA measurements $>16$ weeks before study entry discounted by $50 \%$. All other PSA values, including those on trial, were given equal weighting $($ weight $=1)$. To calculate the mean cohort doubling time, the slopes of individual regression equations were averaged and its inverse multiplied by the log of 2 .

\section{Pharmacokinetic studies}

Blood samples were obtained from all patients during their first course of treatment, at the following time points: pre-dose (within $60 \mathrm{~min}$ of drug administration), post-dose at $30 \mathrm{~min}, 1,2,4,6,24$, 48 and $72 \mathrm{~h}$. In addition, trough samples were taken at days 7,14 and 21. Samples of $10 \mathrm{ml}$ were collected into potassium-EDTA tubes from an indwelling arm vein catheter, and centrifuged at $2000 \mathrm{~g}$ at $4^{\circ} \mathrm{C}$ for $15 \mathrm{~min}$. The plasma was immediately stored at $-80^{\circ} \mathrm{C}$. Urine was collected over a 24 -h period immediately after the first dose on day 1 . The 24 -h urine sample was thoroughly mixed, volume recorded and a 6-ml aliquot was taken and stored at $-80^{\circ} \mathrm{C}$. Samples were analysed for sodium selenate (parent) and its various metabolites using ion chromatography dynamic reaction cell inductively coupled plasma mass spectrometry (Kuo and Jiang, 2008).

\section{Pharmacokinetic analysis}

Pharmacokinetic results were processed according to standard non-compartmental analytical procedures (Win Non Lin v5.2, Pharsight Corporation, Mountain View, CA, USA). The actual times of sample collections were used in the calculations. Parameters measured included $C_{\max }, C_{\min }, T_{\max }$ and $\mathrm{AUC}_{\text {last }}$. AUC was determined by the linear trapezoidal rule, where $A_{U C} C_{\text {last }}$ was the AUC from time zero until the last concentration point. The elimination rate constant, $\mathrm{Kel}\left(\mathrm{h}^{-1}\right)$ was determined by linear regression of a minimum of three points. Half-life $\left(t_{1 / 2}\right)$ was determined according to $t_{1 / 2}=0.693 \mathrm{Kel}^{-1}$.

\section{Statistical evaluations}

All data presented are descriptive and no formal statistical evaluation was performed.

\section{RESULTS}

\section{Patient characteristics}

Between June 2006 and November 2008, a total of 19 patients were enrolled in the study and their clinical characteristics are summarised in Table 1 . The median age was 72 years and the mean PSA was $22 \mu \mathrm{gl}^{-1}$, with an average PSA doubling time of 2.2 months $(1.1-8.9)$. In all, 7 of 19 patients $(36.9 \%)$ had previously received treatment to the primary tumour, three with radical surgery and four with external beam radiotherapy. The remaining 12 patients had androgen deprivation as their only prostate cancer treatment. In all, 10 of 19 patients $(52.6 \%)$ had metastatic bone disease at the time of enrolment.
Table I Patient characteristics

\begin{tabular}{lc}
\hline$n$ & 19 \\
Mean age (range), years & $72(58-78)$ \\
Caucasian, $n(\%)$ & $19(100)$ \\
Mean PSA (range), $\mu \mathrm{gl}^{-1}$ & $22(7.5-245.1)$ \\
Mean PSAdt (range), months & $2.18(1.05-8.94)$ \\
Bone metastases (\%) & $10(52.6)$ \\
Mean haemoglobin (range), $\mathrm{gl}^{-1}$ & $137.6(109-166)$ \\
Mean alkaline phosphatase (range), $\cup \mathrm{I}^{-1}$ & $126.6(59-49 \mid)$ \\
WHO performance status & \\
0 & 15 \\
I & 4 \\
Previous therapy (\%) & \\
Radical prostatectomy & $3(15.8)$ \\
EBRT & $4(21.1)$ \\
Median Gleason score (range) & $8(6-10)$ \\
\hline
\end{tabular}

Abbreviations: EBRT = External beam radiotherapy; PSA = prostate-specific antigen; PSAdt $=$ PSA doubling time; $\mathrm{WHO}=$ World Health Organisation

Table 2 Dose escalation and treatment completion

\begin{tabular}{|c|c|c|c|}
\hline Dose & $\mathbf{N}$ & $\begin{array}{l}\text { Completed } \\
\text { I 2-week } \\
\text { treatment }\end{array}$ & Reason for withdrawal \\
\hline 5 mg per day & I & Yes & \\
\hline 10 mg per day & 1 & Yes & \\
\hline 15 mg per day & I & Yes & \\
\hline $30 \mathrm{mg}$ per day & 1 & Yes & \\
\hline \multirow[t]{3}{*}{30 mg ( 10 mg t.d.s.) } & 3 & No & Disease progression \\
\hline & & Yes & \\
\hline & & Yes & \\
\hline \multirow[t]{2}{*}{45 mg ( 15 mg t.d.s.) } & 2 & Yes & \\
\hline & & Yes & \\
\hline \multirow[t]{6}{*}{60 mg (20 mg t.d.s.) } & 6 & No & Disease progression \\
\hline & & No & Disease progression \\
\hline & & No & Disease progression \\
\hline & & Yes & \\
\hline & & Yes & \\
\hline & & No & $\begin{array}{l}\text { Adverse event } \\
\text { (renal impairment) }\end{array}$ \\
\hline \multirow[t]{4}{*}{90 mg (30 mg t.d.s.) } & 4 & No & DLT (grade 3 fatigue) \\
\hline & & Yes & \\
\hline & & Yes & \\
\hline & & No & $\begin{array}{l}\mathrm{DLT} \text { (grade } 3 \text { diarrhoea and } \\
\text { muscle spasms) }\end{array}$ \\
\hline
\end{tabular}

Abbreviation: $\mathrm{DLT}=$ dose-limiting toxicity.

\section{Safety results}

Of the 19 patients enrolled into the study, 12 patients (63\%) completed the 12-week treatment period (Table 2). In all, 6 of the 12 patients who completed the 12 -week study period continued to receive study drug beyond this time for a total treatment period ranging from 23-28 weeks. Seven patients withdrew from the study drug before completion of the main treatment phase due to disease progression (four patients), unacceptable toxicity (two patients, one with grade 3 fatigue and one with concomitant grade 3 diarrhoea and muscle cramps) or the occurrence of an adverse event (one patient with acute renal impairment)

Table 3 documents the toxicities observed during the study thought to be at least possibly related to sodium selenate. The majority $(76 \%)$ were classified as grade 1, whereas $20 \%$ were classified as grade 2. Five grade 3 toxicities (fatigue, nail disorders, muscle spasms and diarrhoea) were reported by three patients in the $90-\mathrm{mg}$ ( $30 \mathrm{mg}$ t.d.s.) cohort. All of these events resolved, although two of the three patients required that study drug be 
permanently discontinued. No grade 4 events were reported and there were no deaths on study.

Two events considered to be DLTs (grade 3 fatigue, and concomitant grade 3 diarrhoea and intermittent muscle cramps) were reported by two patients in the $90-\mathrm{mg}$ ( $30 \mathrm{mg}$ t.d.s.) cohort. Thus, $60 \mathrm{mg}$ ( $20 \mathrm{mg}$ t.d.s.) was determined to be the MTD. The DRTs were seen in the $60-\mathrm{mg}(20 \mathrm{mg}$ t.d.s.) and $90-\mathrm{mg}(30 \mathrm{mg}$ t.d.s.) cohorts. Fatigue was the most commonly reported DRT.

Table 3 Toxicity experienced by $\geqslant 2$ patients thought to be at least possibly related to the study drug

\begin{tabular}{llcc}
\hline SOC & $\begin{array}{l}\text { Preferred } \\
\text { term }\end{array}$ & $\begin{array}{c}\text { Grade I/2 } \\
(\mathbf{\%})\end{array}$ & $\begin{array}{c}\text { Grade 3 } \\
\mathbf{( \% )}\end{array}$ \\
\hline Gastrointestinal disorders & Constipation & $4(21)$ & $0(0)$ \\
& Diarrhoea & $4(21)$ & $1(5)$ \\
& Nausea & $5(26)$ & $0(0)$ \\
& Retching & $2(10)$ & $0(0)$ \\
& Vomiting & $4(21)$ & $0(0)$ \\
General disorders & Fatigue & $8(42)$ & $1(5)$ \\
Metabolism and nutrition & Decreased & $5(26)$ & $0(0)$ \\
Musculoskeletal and connective tissue & appetite & & \\
disorders & Arthralgia & $2(10)$ & $0(0)$ \\
& Muscle spasms & $8(42)$ & $1(5)$ \\
Nervous system & Myalgia & $3(16)$ & $0(0)$ \\
& Dizziness & $4(21)$ & $0(0)$ \\
& Headache & $3(16)$ & $0(0)$ \\
Skin & Hypoaesthesia & $2(10)$ & $0(0)$ \\
& Lethargy & $5(26)$ & $0(0)$ \\
& Alopecia & $8(42)$ & $0(0)$ \\
& Nail disorders & $5(26)$ & $1(5)$ \\
& Pain of skin & $2(10)$ & $0(0)$ \\
\hline
\end{tabular}

A total of five serious adverse events were reported by five patients, only one of which was considered to have a potential causal relationship to sodium selenate. This was a patient in the 90-mg (30 mg t.d.s.) cohort who presented with an elevated creatinine level of $260 \mathrm{mmoll}^{-1}$ (as compared with $90 \mathrm{mmoll}^{-1}$ at screening) when scheduled biochemistry assessments were performed at the end of the first cycle of treatment. At this visit, the patient was also noted to have decreased bicarbonate levels ( $19 \mathrm{mmoll}^{-1}$ compared with $30 \mathrm{mmol}^{-1}$ at screening), associated with diarrhoea and dehydration. The study drug was stopped immediately and the patient was admitted for investigation of acute renal impairment. Examination of the patient's clinical records revealed the patient had a history of proteinuria, macrohaematuria and underlying kidney disease before commencing sodium selenate. Despite investigation, no cause for the acute deterioration in renal function could be determined, and a contribution of sodium selenate to the existing underlying renal disease cannot be ruled out. Creatinine levels were further elevated to $290 \mathrm{mmoll}^{-1}$ when re-tested 12 weeks after the last study dose, although bicarbonate levels had normalised. The four serious adverse events considered not to be associated with the study drug were episodes of pelvic pain, bowel obstruction, renal colic and lower respiratory tract infection.

Apart from the patient described above, there were no clinically significant laboratory results considered to be potentially related to the study drug, and no patients were found to have vital sign assessments or ECG results that were abnormal and clinically significant.

\section{Pharmacokinetic and metabolic profile}

The PK parameters are detailed in Table 4. Sodium selenate exhibited linear pharmacokinetics, in terms of AUC $v s$ dose, across the dose ranges assessed in this study, with the caveat of small

Table 4 Pharmacokinetic parameters for selenate for all patient cohorts

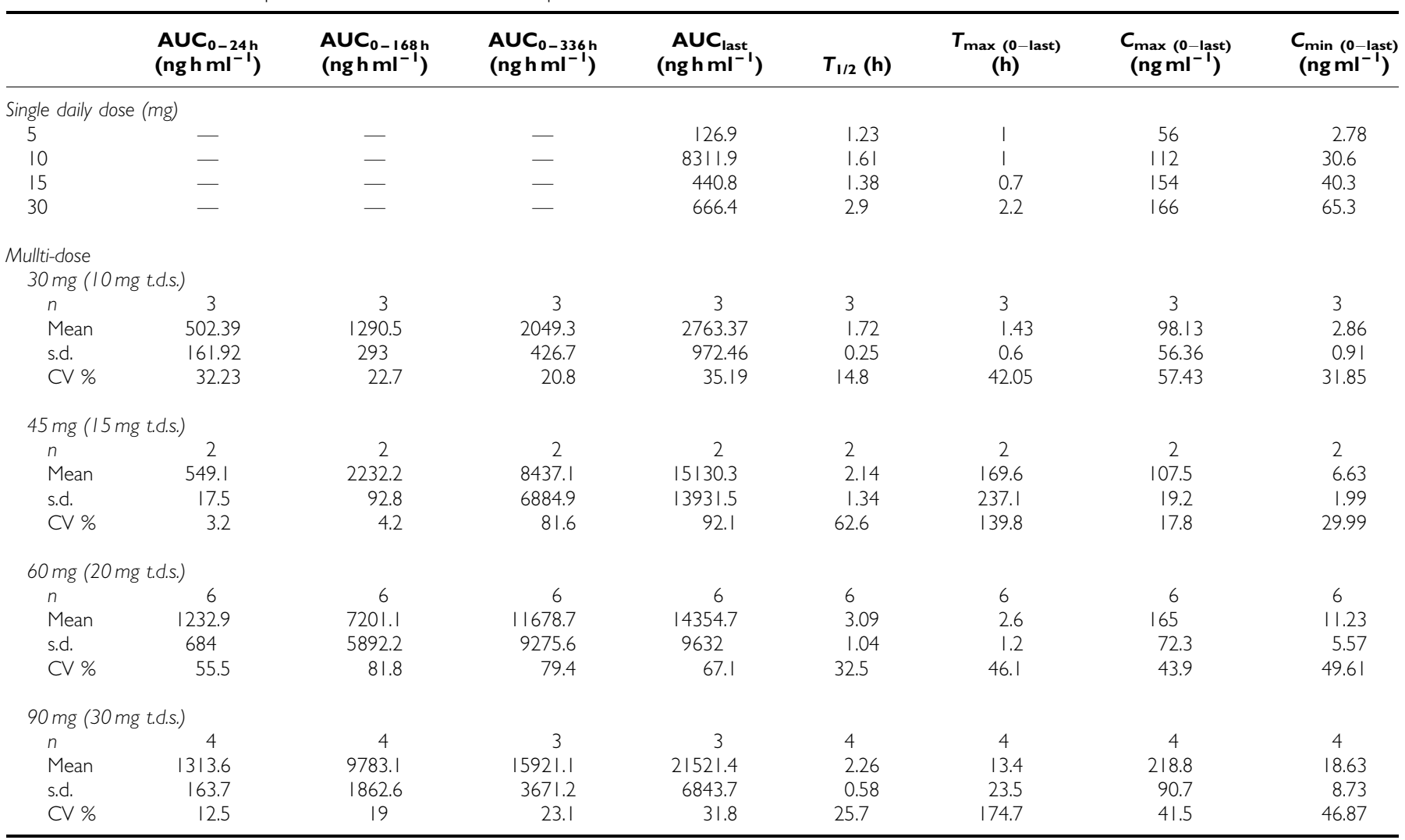

Abbreviation: $\mathrm{CV}=$ coefficient of variance. 
cohort size. The selenate $t_{1 / 2}$ ranged from 1.2 to $2.9 \mathrm{~h}$ in the 5-30 mg single-dose cohorts, respectively. The short half-life necessitated a three times a day daily dosing regimen to maintain adequate blood levels of sodium selenate. Peak selenate plasma concentrations were achieved within $1-4 \mathrm{~h}$ across all dose levels. At the recommended phase II dose, that is, $20 \mathrm{mg}$ t.d.s., the mean $t_{\max }$ was $2.5 \mathrm{~h}$ and the mean $t_{1 / 2}$ was $2.9 \mathrm{~h}$.

The major selenium metabolite identified in plasma was selenite. Its plasma concentrations showed signs of accumulation, with $t_{\max }$ ranging from a mean of $90 \mathrm{~h}$ for the $10 \mathrm{mg}$ t.d.s. cohort to $294 \mathrm{~h}$ for the $30 \mathrm{mg}$ t.d.s. cohort (data not shown). Plasma selenite levels reached steady-state levels by 3 weeks. The ratio of selenite $\mathrm{AUC}_{504}$ $v s$ selenate $\mathrm{AUC}_{504}$ ranged from $1.3 \times$ at $10 \mathrm{mg}$ t.d.s. to $7 \times$ at the $30 \mathrm{mg}$ t.d.s. dose level. Seleno-methionine, seleno-cyanate and methyl selenium species were other significantly minor metabolites identified in plasma. For the t.d.s. dosing cohorts, $16-37 \%$ of total daily dose of selenium was recovered in the 24 -h urine. Selenate was the major selenium species identified in the $24-\mathrm{h}$ urine accounting for $10-24 \%$ (based on the mean for each t.d.s. dosing cohort) of total daily selenium administered. Selenomethionine and miscellaneous methyl selenium species were also major selenium compounds identified in 24-h urine; however, levels varied significantly across the patients in the t.d.s. dosing cohorts. Selenite was barely detectable in the urine, being $<0.3 \%$ of total daily dose.

\section{Efficacy}

Although the study was not designed to determine clinical efficacy, PSA was monitored throughout the trial as a surrogate marker of tumour response. The percentage change in PSA from baseline for each individual patient is shown in Figure 1. One patient from the 60-mg (20 mg t.d.s., patient 14) cohort experienced a significant PSA decline (maximal change in PSA from baseline -57.4\%) that was maintained for 11 weeks and two patients (both from the 30$\mathrm{mg}$ (10 mg t.d.s.) cohort) had disease stabilisation lasting 28 and 41 weeks, respectively. For those patients who completed the initial 12 -week evaluation $(n=12)$, median time to progression was 14.2 weeks $(13.1-41.1)$

Mean doubling time increased from 2.2 months $(1.1-9.0)$ before trial to 3.9 months $(1.1$ to -2.0$)$ during the main treatment phase (Figure 2).

\section{DISCUSSION}

The management of metastatic prostate cancer has changed significantly in the past decade. Cytotoxic chemotherapy with docetaxel is a standard of care in castrate-resistant disease and new therapies, including hormone agents such as abiraterone, show promising activity. However, no current therapy is curative and novel therapies are still needed. Most recently, many clinical trials have focussed on anti-angiogenic agents targeting critical kinases.

We have previously identified that the specific seleniumcontaining compound, sodium selenate, significantly boosts the activity of the protein phosphatase PP2A, inhibits VEGF-induced growth and survival signalling in endothelial cells, and impedes tumour neovascularisation.

This 'first-in-human' study examined the use of sodium selenate in men with CRPC before they received cytotoxic chemotherapy. Sodium selenate was well tolerated at doses up to $45 \mathrm{mg}$ per day t.d.s. and the MTD was attained at a dose of $60 \mathrm{mg}$ per day t.d.s. DLT was fatigue and diarrhoea, while other frequently reported adverse events included, nail disorders, muscle spasms, alopecia and nausea. Some of the side effects observed at the higher doses, in particular the fatigue, diarrhoea, alopecia and vomiting are perhaps attributable to the accumulation of the inorganic metabolite selenite, a selenium compound with marked cytotoxic

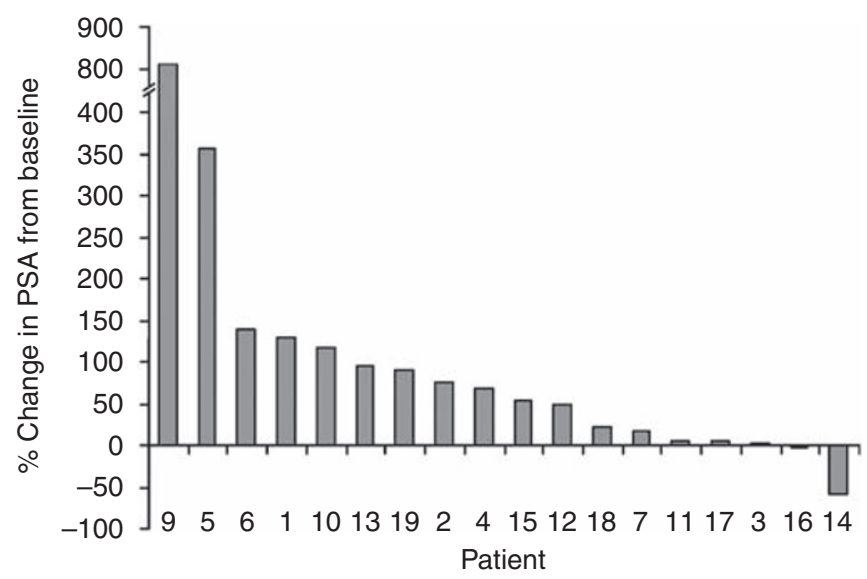

Figure I Waterfall plot of percentage change in prostate-specific antigen (PSA) from baseline to the end of the 12-week evaluation period (or withdrawal if earlier) for each patient.

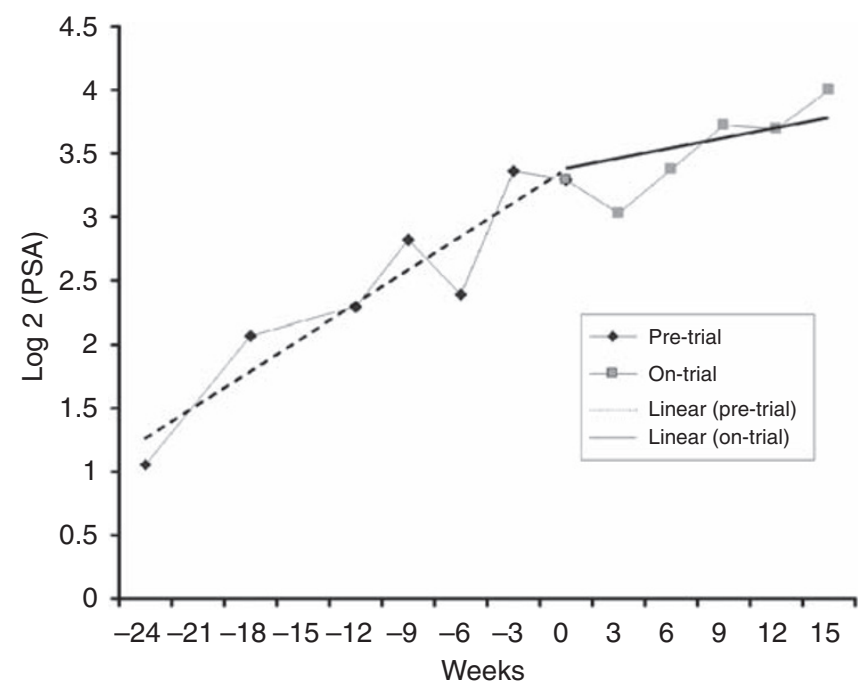

Figure 2 Prostate-specific antigen (PSA) doubling time before trial and during the main treatment phase.

properties and a very different pharmacokinetic profile compared with selenate, as confirmed in this study ( $\mathrm{Lu}$ et al, 2009).

The study was not designed to evaluate the efficacy of sodium selenate. However, one PSA response was observed and two other patients appeared to have some stabilisation of disease. This modest activity is consistent with single-agent studies of other anti-angiogenic agents, including bevacizumab, sorafenib and sunitinib (Reese et al, 2001; Chi et al, 2008; Dror Michaelson et al, 2009).

On the bases of these observations, it would be reasonable to question the importance of angiogenesis to the development of prostate cancer. However, the continual development of a new vascular network is required to support the growth and increase in size of all solid tumours. The density of the vascular network that is established in any given tumour might reasonably be expected to reflect the growth, progression and metastatic potential of that tumour (Folkman, 1971), and indeed, tumour vascularity as measured by MVD has been shown to be a prognostic factor in a range of solid malignancies including that of prostate cancer (Weidner et al, 1991; Weidner, 1993; Weidner and Folkman, 1996; Strohmeyer et al, 2000). 
Most of the evidence linking angiogenesis with prostate cancer progression has come from studies evaluating total MVD in prostate tumour samples, which almost invariably demonstrate a positive correlation with other histological features of tumour aggression and/or adverse clinical outcomes. However, as previously proposed, MVD may be poor surrogate of a tumour angiogenic potential, rather more accurately reflects tissue metabolic activity (Hlatky et al, 2002). Recent findings in renal cell carcinoma suggest that the relative degree of vessel differentiation within tumours correlates more closely with angiogenic activity than total MVD, with high densities of undifferentiated vessels significantly associated with higher tumour grades and shorter patient survival, in contrast to high densities of differentiated, vessel which correlated significantly with lower tumour grade and longer survival (Yao et al, 2007). Similar findings have been reported in prostate cancer samples, with significantly higher levels of proliferating immature tumour vessels identified in castration-resistant or metastatic tissue compared to organ confined cancers or benign tissue (Gravdal et al, 2009). More importantly, higher levels of immature vessels in radical prostatectomy specimens were a strong and independent predictor of biochemical failure, clinical recurrence or the development of bony metastases in multivariate analyses, confirming the clinical importance of angiogenesis in prostate cancer progression.

The expectation that single-agent anti-angiogenic therapy will result in the same type of clinical responses that are observed with traditional cytotoxic agents is based on the assumption that either the tumour itself, or the vascular network that supports it, is critically dependent on the signalling pathway inhibited. However, with the notable exception of clear cell renal carcinoma (CCRCC), significant or sustained responses are rarely observed with singleagent therapy. This clinical difference may simply reflect the strength of angiogenic drive within different tumour types, as renal cancers, together with glioblastomas, have the highest rates of endothelial cell proliferation and levels of immature blood vessels of tumour types studied, certainly significantly higher than prostate cancer, and thus may be more intrinsically sensitive to angiogenic inhibition (Eberhard et al, 2000). In addition, given the high frequency of VHL mutations in CCRCC, angiogenesis may be much more dependent on VEGF signalling than in prostate cancer, in which multiple redundant pathways have been implicated (Gravdal et al, 2006; Kim et al, 2006; Bergers and Hanahan, 2008; Yang et al, 2008). In future studies, the identification of prostate

\section{REFERENCES}

Ahmed Z, Bicknell R (2009) Angiogenic signalling pathways. Methods Mol Biol 467: 3-24

Aragon-Ching JB, Dahut WL (2009) VEGF inhibitors and prostate cancer therapy. Curr Mol Pharmacol 2: 161-168

Baade PD, Coory MD, Aitken JF (2004) International trends in prostatecancer mortality: the decrease is continuing and spreading. Cancer Causes Control 15: 237-241

Bergers G, Hanahan D (2008) Modes of resistance to anti-angiogenic therapy. Nat Rev Cancer 8: $592-603$

Bok RA, Halabi S, Fei DT, Rodriquez CR, Hayes DF, Vogelzang NJ, Kantoff P, Shuman MA, Small EJ (2001) Vascular endothelial growth factor and basic fibroblast growth factor urine levels as predictors of outcome in hormone-refractory prostate cancer patients: a cancer and leukemia group B study. Cancer Res 61: 2533-2536

Chi KN, Ellard SL, Hotte SJ, Czaykowski P, Moore M, Ruether JD, Schell AJ, Taylor S, Hansen C, Gauthier I, Walsh W, Seymour L (2008) A phase II study of sorafenib in patients with chemo-naive castration-resistant prostate cancer. Ann Oncol 19: 746-751

Corcoran NM, Martin D, Hutter-Paier B, Windisch M, Nguyen T, Nheu L, Sundstrom LE, Costello AJ, Hovens CM (2010) Sodium selenate specifically activates PP2A phosphatase, dephosphorylates tau and cancer patients most likely to respond to anti-angiogenic therapies, either by measuring levels of immature vessels in pretreatment biopsies, or by global markers of vessel turnover such as with circulating endothelial cells or their progenitors, may be important (Georgiou et al, 2008).

Despite their lack of single-agent activity, anti-angiogenic agents have shown favourable effects in boosting the efficacy of standard chemotherapy regimens in different tumour types (Ellis and Hicklin, 2008). Interestingly, this effect may be independent of a direct effect on new blood vessel formation, but more reliant on a relative normalisation of the vascular network and blood flow within tumours, improving delivery of chemotherapy. Disappointingly however, the recently completed but yet to be reported CALBG phase III study of docetaxel \pm bevacizumab (CALGB 90401) in men with CRPC failed to demonstrate an improvement in survival compared with chemotherapy alone (http://www. roche.com/media/media_releases/med-cor-2010-03-12.htm). As only the VEGF signalling pathway was targeted, it is possible that combining anti-angiogenic agents with different mechanisms of action can overcome the inherent redundancy in angiogenic signalling in prostate cancer, and improve clinical response rates. This is supported by recently published phase II data exploring the combination of docetaxel, bevacizumab, thalidomide and prednisone, with $90 \%$ of patients experiencing a PSA decline of $\geqslant 50 \%$ (Ning et al, 2010).

Further exploration of sodium selenate as a single-agent in CRPC is not warranted; however, its combination with conventional cytotoxics in particular docetaxel, as well as with other anti-angiogenics with different modes of action is worthy of further exploration.

\section{ACKNOWLEDGEMENTS}

We acknowledge the assistance of the following people in the initial design and conduct of the studies: Grant Morley, Patricia Bugeja and Helen Crowe. This work was financially supported by Velacor Therapeutics Pty Ltd in their role as sponsor.

\section{Conflict of interest}

$\mathrm{CMH}$ has received payment as a scientific advisor to Velacor Therapeutics. AJC is a non paid director of Velacor Therapeutics. reverses memory deficits in an Alzheimer's disease model. J Clin Neurosci 17: 1025-1033

Corcoran NM, Najdovska M, Costello AJ (2004) Inorganic selenium retards progression of experimental hormone refractory prostate cancer. J Urol 171: $907-910$

Daskivich TJ, Regan MM, Oh WK (2006) Prostate specific antigen doubling time calculation: not as easy as 1, 2, 4. J Urol 176: 1927-1937

Dror Michaelson M, Regan MM, Oh WK, Kaufman DS, Olivier K, Michaelson SZ, Spicer B, Gurski C, Kantoff PW, Smith MR (2009) Phase II study of sunitinib in men with advanced prostate cancer. Ann Oncol 20: $913-920$

Eberhard A, Kahlert S, Goede V, Hemmerlein B, Plate KH, Augustin HG (2000) Heterogeneity of angiogenesis and blood vessel maturation in human tumors: implications for antiangiogenic tumor therapies. Cancer Res 60: $1388-1393$

Ellis LM, Hicklin DJ (2008) VEGF-targeted therapy: mechanisms of antitumour activity. Nat Rev Cancer 8: 579-591

Folkman J (1971) Tumor angiogenesis: therapeutic implications. N Engl $J$ Med 285: $1182-1186$

George DJ, Halabi S, Shepard TF, Vogelzang NJ, Hayes DF, Small EJ, Kantoff PW (2001) Prognostic significance of plasma vascular endothelial 
growth factor levels in patients with hormone-refractory prostate cancer treated on Cancer and Leukemia Group B 9480. Clin Cancer Res 7: $1932-1936$

Georgiou HD, Namdarian B, Corcoran NM, Costello AJ, Hovens CM (2008) Circulating endothelial cells as biomarkers of prostate cancer. Nat Clin Pract Urol 5: $445-454$

Gravdal K, Halvorsen OJ, Haukaas SA, Akslen LA (2006) Expression of bFGF/FGFR-1 and vascular proliferation related to clinicopathologic features and tumor progress in localized prostate cancer. Virchows Arch 448: $68-74$

Gravdal K, Halvorsen OJ, Haukaas SA, Akslen LA (2009) Proliferation of immature tumor vessels is a novel marker of clinical progression in prostate cancer. Cancer Res 69: 4708-4715

Halvorsen OJ, Haukaas S, Hoisaeter PA, Akslen LA (2000) Independent prognostic importance of microvessel density in clinically localized prostate cancer. Anticancer Res 20: 3791-3799

Hlatky L, Hahnfeldt P, Folkman J (2002) Clinical application of antiangiogenic therapy: microvessel density, what it does and doesn't tell us. J Natl Cancer Inst 94: 883-893

Ivy SP, Wick JY, Kaufman BM (2009) An overview of small-molecule inhibitors of VEGFR signaling. Nat Rev Clin Oncol 6: 569-579

Jemal A, Siegel R, Ward E, Hao Y, Xu J, Thun MJ (2009) Cancer statistics, 2009. CA Cancer J Clin 59: 225-249

Kim SJ, Uehara H, Yazici S, Busby JE, Nakamura T, He J, Maya M, Logothetis C, Mathew P, Wang X, Do KA, Fan D, Fidler IJ (2006) Targeting platelet-derived growth factor receptor on endothelial cells of multidrug-resistant prostate cancer. J Natl Cancer Inst 98: 783-793

Kim YS, Ahn KH, Kim SY, Jeong JW (2009) Okadaic acid promotes angiogenesis via activation of hypoxia-inducible factor-1. Cancer Lett 276: $102-108$

Kuo CY, Jiang SJ (2008) Determination of selenium and tellurium compounds in biological samples by ion chromatography dynamic reaction cell inductively coupled plasma mass spectrometry. J Chromatogr A 1181: $60-66$

Lu J, Berndt C, Holmgren A (2009) Metabolism of selenium compounds catalyzed by the mammalian selenoprotein thioredoxin reductase. Biochim Biophys Acta 1790: $1513-1519$

Mucci LA, Powolny A, Giovannucci E, Liao Z, Kenfield SA, Shen R, Stampfer MJ, Clinton SK (2009) Prospective study of prostate tumor angiogenesis and cancer-specific mortality in the health professionals follow-up study. J Clin Oncol 27: 5627-5633

Ning YM, Gulley JL, Arlen PM, Woo S, Steinberg SM, Wright JJ, Parnes HL, Trepel JB, Lee MJ, Kim YS, Sun H, Madan RA, Latham L, Jones E,
Chen CC, Figg WD, Dahut WL (2010) Phase II trial of bevacizumab, thalidomide, docetaxel, and prednisone in patients with metastatic castration-resistant prostate cancer. J Clin Oncol 28: 2070-2076

Reese DM, Fratesi P, Corry M, Novotny W, Holmgren E, Small E (2001) A phase II trial of humanized anti-vascular endothelial growth factor antibody for the treatment of androgen-independent prostate cancer. Prostate J 3: $65-70$

Revelos K, Petraki C, Scorilas A, Stefanakis S, Malovrouvas D, Alevizopoulos N, Kanellis G, Halapas A, Koutsilieris M (2007) Correlation of androgen receptor status, neuroendocrine differentiation and angiogenesis with time-to-biochemical failure after radical prostatectomy in clinically localized prostate cancer. Anticancer Res 27: $3651-3660$

Scher HI, Halabi S, Tannock I, Morris M, Sternberg CN, Carducci MA, Eisenberger MA, Higano C, Bubley GJ, Dreicer R, Petrylak D, Kantoff P, Basch E, Kelly WK, Figg WD, Small EJ, Beer TM, Wilding G, Martin A, Hussain M (2008) Design and end points of clinical trials for patients with progressive prostate cancer and castrate levels of testosterone: recommendations of the Prostate Cancer Clinical Trials Working Group. J Clin Oncol 26: $1148-1159$

Strohmeyer D, Rossing C, Strauss F, Bauerfeind A, Kaufmann O, Loening S (2000) Tumor angiogenesis is associated with progression after radical prostatectomy in pT2/pT3 prostate cancer. Prostate 42: $26-33$

Weidner N (1993) Tumor angiogenesis: review of current applications in tumor prognostication. Semin Diagn Pathol 10: $302-313$

Weidner N, Folkman J (1996) Tumoral vascularity as a prognostic factor in cancer. Important Adv Oncol 167-190

Weidner N, Semple JP, Welch WR, Folkman J (1991) Tumor angiogenesis and metastasis-correlation in invasive breast carcinoma. $N$ Engl J Med 324: $1-8$

Yang F, Strand DW, Rowley DR (2008) Fibroblast growth factor-2 mediates transforming growth factor-beta action in prostate cancer reactive stroma. Oncogene 27: 450-459

Yao X, Qian CN, Zhang ZF, Tan MH, Kort EJ, Yang XJ, Resau JH, Teh BT (2007) Two distinct types of blood vessels in clear cell renal cell carcinoma have contrasting prognostic implications. Clin Cancer Res 13: $161-169$

Young MR (2004) Tumor-derived prostaglandin E2 and transforming growth factor-beta stimulate endothelial cell motility through inhibition of protein phosphatase-2A and involvement of PTEN and phosphatidylinositide 3-kinase. Angiogenesis 7: 123-131

Young MR, Kolesiak K, Meisinger J (2002) Protein phosphatase-2A regulates endothelial cell motility and both the phosphorylation and the stability of focal adhesion complexes. Int J Cancer 100: 276-282 